trial, individuals in complete or partial remission were randomized to rituximab maintenance therapy $\left(375 \mathrm{mg} / \mathrm{m}^{2}\right.$ intravenously once every 3 months for $\leq 2$ years) or observation. Rituximab maintenance therapy significantly improved PFS (51.5 months, vs 14.9 months with observation; $P<0.001)$ and 3 -year overall survival $(P=0.011)$ from second randomization. PFS improved with rituximab maintenance whether patients had undergone CHOP $(P<0.001)$ or $\mathrm{R}-\mathrm{CHOP}$ $(P=0.004)$ induction.

Further investigation into the optimum regimen is required, as is longer follow-up to determine whether the benefit of this regimen continues; however, these results are promising.

Original article van Oers MHJ et al. (2006) Rituximab maintenance improves clinical outcome of relapsed/resistant follicular non-Hodgkin lymphoma in patients both with and without rituximab during induction: results of a prospective randomized phase 3 intergroup trial. Blood 108: 3295-3301

\section{MRI proves accurate in detecting cervical involvement in endometrial cancer}

Radical hysterectomy for endometrial cancer with cervical involvement markedly increases 5-year survival compared with simple hysterectomy; however, the radical procedure is associated with higher levels of comorbidity. Results from a number of studies indicate that preoperative $\mathrm{MRI}$ could be useful in detecting cervical involvement in endometrial cancer, but the sensitivity and specificity of this technique are unclear.

To determine the diagnostic accuracy of MRI in detecting cervical invasion by endometrial cancer, Nagar et al. carried out a retrospective study of 135 women diagnosed with endometrial cancer. Overall, MRI had a sensitivity of $72 \%$ (95\% Cl 59.8-81.8\%) and a specificity of $93.2 \%$ (95\% Cl 85.1-97.1\%) for the detection of any cervical involvement in endometrial cancer. The false-positive rate for cervical invasion was $6.8 \%(95 \% \mathrm{Cl} 2.9-14.9 \%)$ and the falsenegative rate was $28 \%(95 \% \mathrm{Cl} 18.2-40.2 \%)$. The sensitivity and specificity of MRI for detecting cervical stromal invasion alone were 84.4\% (95\% Cl 68.2-93.1\%) and 87.4\% (95\% Cl 79.6$92.5 \%)$, respectively. The false-positive rate for cervical stromal involvement was $12.6 \%$ (95\% $\mathrm{Cl} 7.5-20.4 \%)$ and the false-negative rate was $15.4 \%$ (95\% Cl 6.9-31.8\%).
On the basis of these results, the authors conclude that MRI can accurately predict cervical involvement in endometrial cancer and could help clinicians to offer the most appropriate surgical procedure to women presenting with the disease.

Original article Nagar H et al. (2006) The diagnostic accuracy of magnetic resonance imaging in detecting cervical involvement in endometrial cancer. Gynecol Oncol 103: 431-434

\section{Sentinel lymph-node biopsy of the internal mammary chain could aid breast cancer staging}

Although axillary sentinel lymph-node (SLN) biopsy is commonly used to provide staging information for breast cancer, internal mammary chain (IMC) SLN removal is not routinely practiced. In a study involving 741 women diagnosed with breast cancer, Carcoforo et al. examined the incidence of IMC SLNs, and assessed the effect of IMC SLN biopsy on tumor staging and subsequent choice of therapeutic strategy.

Preoperative lymphoscintigraphy revealed 107 IMC hotspots; gamma probe scans in the operating room subsequently confirmed 72 IMC SLNs in 65 patients. Of these 65 patients, 10 later tested positive for IMC SLNs at pathologic examination. Notably, only 1 of these 10 patients also had axillary metastasis. Overall, $9 \%$ of tumors showed IMC drainage. Although IMC SLN biopsy has the potential to increase morbidity compared with axillary node biopsy, only two complications, both involving minor pleural tears, were documented in this study. In total, 10 patients had their staging changed (1 patient from pN1a to $\mathrm{pN} 1 \mathrm{c}$ and 9 patients from $\mathrm{pN} 0$ to $\mathrm{pN} 1$ ). In addition, nine patients had their therapy changed to include adjuvant radiotherapy to the internal mammary region.

While calling for further trials to determine whether IMC SLN biopsy improves survival rates, the authors conclude that IMC SLN biopsy is a safe procedure that might assist in the accurate staging of patients with breast cancer.

\footnotetext{
Original article Carcoforo P et al. (2006) Clinical and therapeutic importance of sentinel node biopsy of the internal mammary chain in patients with breast cancer: a single-center study with long-term follow-up. Ann Surg Oncol 13: 1338-1343
} 